\title{
Almost Sure Limit Inferior for Increments of Stable Subordinators*
}

\author{
Kokkada Vidyalaxmi, Korakote Nagaraj Ravi Prakash, Gooty Divanji ${ }^{\#}$ \\ Department of Studies in Statistics, University of Mysore, Manasagangothri, Mysore, India \\ Email: vidyalaxmi.k@gmail.com, raviprakashmaiya@gmail.com, "gootydivan@yahoo.co.in
}

Received 22 June 2014; revised 2 August 2014; accepted 12 September 2014

Copyright (C) 2014 by authors and OALib.

This work is licensed under the Creative Commons Attribution International License (CC BY).

http://creativecommons.org/licenses/by/4.0/

(c) (i) Open Access

\section{Abstract}

Let $\{X(t), 0 \leq t<\infty\}$ be a sequence of completely asymmetric stable process or stable subordinators defined on a common probability space $(\Omega, \mathfrak{I}, \boldsymbol{P})$. In this paper, for proper selection of norming constants, we study almost sure limit inferior for increments of stable subordinators of geometrically increasing subsequences. Also we obtain similar results to delayed sums and study the existence of moments for boundary crossing random variables.

\section{Keywords}

Increments, Stable Subordinators, Law of Iterated Logarithm, Delayed Sums and Subsequences

Subject Areas: Mathematical Statistics, Probability Theory

\section{Introduction}

Let $\{X(t), 0 \leq t<\infty\}$ be a completely asymmetric stable process or subordinators defined on a common probability space $(\Omega, \mathfrak{I}, P)$. Let $a_{t}, t>0$, be a non-negative valued function of $t$ such that 1$) 0<a_{t} \leq t$ 2) $a_{t} \rightarrow \infty$, as $t \rightarrow \infty$ and 3) $\left(a_{t} / t\right) \downarrow 0$, as $t \rightarrow \infty$. Let $Y(t)=X\left(t+a_{t}\right)-X(t), t>0$ and $Y(0)=0$. Define $\beta(t)=\theta_{\alpha} a_{t}^{\frac{1}{\alpha}}\left(\log \frac{t}{a_{t}}+\log \log t\right)^{\frac{\alpha-1}{\alpha}}$, where $\theta_{\alpha}=(B(\alpha))^{\frac{1-\alpha}{\alpha}}$ and $B(\alpha)=(1-\alpha) \alpha^{\frac{\alpha-1}{\alpha}}\left(\cos \left(\frac{\pi \alpha}{2}\right)\right)^{\frac{1}{\alpha-1}}$, where $0<\alpha<1$. Observe that the process has the property that $t^{-1 / \alpha} X(t)$ and $X(1)$ are identically distributed.

A real valued increasing process $\{X(t), t>0\}$ with stationary independent increments is called a subordi-

*AMS Subject Classification: 60F15 and 60G17.

"Corresponding author.

How to cite this paper: Vidyalaxmi, K., Prakash, K.N.R. and Divanji, G. (2014) Almost Sure Limit Inferior for Increments of Stable Subordinators. Open Access Library Journal, 1: e812. http://dx.doi.org/10.4236/oalib.1100812 
nator. At any fixed $t$, the characteristic function of $X(t)$ can be given by, $\log f_{t}(u)=t\left(\int_{0}^{\infty}\left(\mathrm{e}^{i u x}-1\right) \mathrm{d} H(x)\right)$, $t \in[0, \infty)$, where $H(x)$ is a spectral function which satisfies $\int_{0}^{\infty} \frac{x}{1+x} \mathrm{~d} H(x)<\infty$. When $H(x)=-\frac{C}{x^{\alpha}}$, $C>0,0<\alpha<1$, the subordinator becomes a stable subordinator with characteristic exponent $\alpha, 0<\alpha<1$. For a class of stable subordinators or asymmetric stable processes see Mijnheer ([4], Theorem 5.2.1 on Page 48).

Vasudeva and Divanji ([7], Theorem) have obtained the following limit inferior for the increments of stable subordinators. Under certain conditions on $a_{t}$, it was shown that $\operatorname{Liminf}_{t \rightarrow \infty} \frac{Y(t)}{\beta(t)}=1$ a.s.

The LIL for partial sums $S_{n}$ of r.v.s with unit variance states that for $a_{n}=\sqrt{2 n \log \log n}$, we have

$$
\operatorname{Lim} \sup _{n \rightarrow \infty}(\inf ) \frac{S_{n}}{a_{n}}=1(-1) \text { a.s. }
$$

Allan Gut ([1], Theorem 2.1 and Theorem 2.2) and Rainer Schwabe and Allan Gut ([6], Theorem 3.2) have studied the behavior of LIL for subsequences $\left\{n_{k}, k \geq 1\right\}$. It depends how fast $n_{k}$ tends to infinity and whether we can take $a_{n}=\sqrt{2 n \log \log n}$.

Kyo Shin Hwang, Yong Kab Choi and Jong Soo Jung ([2], Theorems 1-3) studied this subsequence principle for increments of Gaussian processes in obtaining limit superior. In this work we study an almost sure limit inferior behaviour for increments of stable subordinators for proper selection of subsequences and extended to delayed sums. We notice that for different selections of subsequence, the dividing term in limit inferior changes with $a_{t}$.

We present a known lemma and results for increments of stable subordinators in next section. In the last section, we give the parallel results for delayed sums and study the existence of moments for boundary crossing random variables.

\section{LIL for Increments of Stable Subordinators}

In this section first we state needed lemma and give our main results.

\section{Lemma}

Let $X_{1}$ be a positive stable r.v. with characteristic function

$$
\begin{aligned}
E\left(\exp \left\{i u X_{1}\right\}\right)= & \exp \left\{-|u|^{\alpha}\left(1-\frac{i u}{|u|} \tan \left(\frac{\pi \alpha}{2}\right)\right)\right\}, 0<\alpha<1 . \text { Then, as } x \rightarrow 0, \\
& P\left(X_{1} \leq x\right) \simeq \frac{x^{\frac{\alpha}{2(1-\alpha)}}}{\sqrt{2 \pi \alpha B(\alpha)}} \exp \left\{-B(\alpha) x^{\frac{\alpha}{\alpha-1}}\right\},
\end{aligned}
$$

where

$$
B(\alpha)=(1-\alpha) \alpha^{\frac{\alpha-1}{\alpha}}\left(\cos \left(\frac{\pi \alpha}{2}\right)\right)^{\frac{1}{\alpha-1}}
$$

\section{Proof}

See Vasudeva and Divanji [7] or Mijnheer [4].

\section{Theorem 2.1}

Let $a_{t}, t>0$, be a non-decreasing function of $t$ such that 1) $\left.0<a_{t} \leq t, 2\right) a_{t} \rightarrow \infty$, as $t \rightarrow \infty$ and 3) $\frac{a_{t}}{t} \downarrow 0$, as $t \rightarrow \infty$. Let $\left(t_{k}\right)$ be an increasing sequence of positive integers such that

$$
\underset{k \rightarrow \infty}{\operatorname{Limsup}} \frac{t_{k+1}-t_{k}}{a_{t_{k}}}<1 \text {. }
$$

Then 


$$
\operatorname{Liminf}_{k \rightarrow \infty} \frac{Y\left(t_{k}\right)}{\beta\left(t_{k}\right)}=\varepsilon^{*} \text { a.s. , }
$$

where

$$
\varepsilon^{*}=\inf \left\{\varepsilon>0: \sum_{k=k_{0}}^{\infty}\left(g\left(t_{k}\right)\right)^{-\varepsilon^{-\lambda}}<\infty\right\}, g\left(t_{k}\right)=\left(\frac{t_{k}}{a_{t_{k}}} \log t_{k}\right) \text { and } \lambda=\frac{\alpha}{\alpha-1}<0,0<\alpha<1 .
$$

\section{Proof}

Equivalently, we show that for any given $\varepsilon_{1}>0$, as $k \rightarrow \infty$,

$$
P\left(Y\left(t_{k}\right) \leq\left(\varepsilon^{*}+\varepsilon_{1}\right) \beta\left(t_{k}\right) \text { i.o }\right)=1
$$

and

$$
P\left(Y\left(t_{k}\right) \leq\left(\varepsilon^{*}-\varepsilon_{1}\right) \beta\left(t_{k}\right) \text { i.o }\right)=0
$$

The condition (2.1) implies that $t_{k+1}<t_{k}+a_{t_{k}}$, for large $k$ and by Mijnheer [5], we have

$$
P\left(Y\left(t_{k}\right) \leq\left(\varepsilon^{*}+\varepsilon_{1}\right) \beta\left(t_{k}\right)\right)=P\left(X(1) \leq \frac{\left(\varepsilon^{*}+\varepsilon_{1}\right) \beta\left(t_{k}\right)}{a_{t_{k}}^{\frac{1}{\alpha}}}\right)
$$

Observe that $\frac{\left(\varepsilon^{*}+\varepsilon_{1}\right) \beta\left(t_{k}\right)}{a_{t_{k}}^{\frac{1}{\alpha}}}=\left(\varepsilon^{*}+\varepsilon_{1}\right) \theta_{\alpha}\left(\log \frac{t_{k}}{a_{t_{k}}}+\log \log t_{k}\right)^{\frac{\alpha-1}{\alpha}}$ taken as $x$, in the above lemma, one can find a $k_{1}$ and some constant $C_{1}$, such that for all $k \geq k_{1}$,

$$
P\left(X(1) \leq \frac{\left(\varepsilon^{*}+\varepsilon_{1}\right) \beta\left(t_{k}\right)}{a_{t_{k}}^{\frac{1}{\alpha}}}\right) \geq \frac{C_{1}}{\left(\log g\left(t_{k}\right)\right)^{\frac{1}{2}}} \exp \left\{-\left(\varepsilon^{*}+\varepsilon_{1}\right)^{\frac{\alpha}{\alpha-1}} \log g\left(t_{k}\right)\right\},
$$

where $g\left(t_{k}\right)=\left(\frac{t_{k}}{a_{t_{k}}} \log t_{k}\right)$. Notice that from the definition of $\varepsilon^{*}$, we have $\varepsilon^{*} \geq 1$ implies that there exists $\varepsilon_{2}$ $(>0)$ small such that $\left(\varepsilon^{*}+\varepsilon_{1}\right)^{\frac{\alpha}{\alpha-1}}<\left(1-\varepsilon_{2}\right)<1$. Hence $P\left(X(1) \leq \frac{\left(\varepsilon^{*}+\varepsilon_{1}\right) \beta\left(t_{k}\right)}{a_{t_{k}}^{\frac{1}{\alpha}}}\right) \geq \frac{C_{1}}{\left(\log g\left(t_{k}\right)\right)^{\frac{1}{2}}\left(g\left(t_{k}\right)\right)^{\left(1-\varepsilon_{2}\right)}}$. Let $l_{k}=\frac{t_{k}}{a_{t_{k}}}$ and $m_{k}=\log t_{k}$. Since $\frac{a_{t_{k}}}{t_{k}} \downarrow 0$, as $k \rightarrow \infty, l_{\mathrm{k}}$ is non-decreasing and $m_{k} \rightarrow \infty$, as $k \rightarrow \infty$, one can find a constant $k_{2} \geq k_{1}$ such that $\frac{l_{k}^{\varepsilon_{2}} m_{k}^{\varepsilon_{2}}}{\left(\log l_{k} m_{k}\right)^{\frac{1}{2}}} \geq 1$, whenever $k \geq k_{2}$. By condition (2.1), for all $k \geq k_{2}$, we therefore have,

$$
P\left(X(1) \leq \frac{\left(\varepsilon^{*}+\varepsilon_{1}\right) \beta\left(t_{k}\right)}{a_{t_{k}}^{\frac{1}{\alpha}}}\right) \geq C_{1} \frac{t_{k+1}-t_{k}}{t_{k} \log t_{k}} .
$$

We know that $\int_{t_{k_{2}}}^{\infty} \frac{\mathrm{d} t}{t \log t} \leq \sum_{k=k_{2}}^{\infty} \frac{t_{k+1}-t_{k}}{t_{k} \log t_{k}}$ and $\int_{t_{k_{2}}}^{\infty} \frac{\mathrm{d} t}{t \log t}=\infty$. Hence from (2.4) and (2.5), we get, 


$$
\sum_{k=k_{2}}^{\infty} P\left(Y\left(t_{k}\right) \leq\left(\varepsilon^{*}+\varepsilon_{1}\right) \beta\left(t_{k}\right)\right)=\infty .
$$

Condition (2.1) implies that $t_{k+1} \leq t_{k}+a_{t_{k}}$, for large $k$ one can observe that $Y\left(t_{k}\right)$ 's are mutually independent and hence by Borel zero-one lemma, we have,

$$
P\left(Y\left(t_{k}\right) \leq\left(\varepsilon^{*}+\varepsilon_{1}\right) \beta\left(t_{k}\right) \text { i.o }\right)=1,
$$

which establishes (2.2).

We now complete the proof by showing that, for any $\varepsilon_{1} \in(0,1), P\left(Y\left(t_{k}\right) \leq\left(\varepsilon^{*}-\varepsilon_{1}\right) \beta\left(t_{k}\right)\right.$ i.o $)=0$. By condition (2.1), we have $t_{k+1} \leq t_{k}+a_{t_{k}}$, for large $k$ and from Mijnheer [5], one can find a $k_{3}$ such that for all $k \geq k_{3}$,

$$
P\left(Y\left(t_{k}\right) \leq\left(\varepsilon^{*}-\varepsilon_{1}\right) \beta\left(t_{k}\right) \text { i.o }\right)=P\left(X\left(t_{k}+a_{t_{k}}\right)-X\left(t_{k}\right) \leq\left(\varepsilon^{*}-\varepsilon_{1}\right) \beta\left(t_{k}\right) \text { i.o }\right)
$$

Hence in order to prove (2.3), it is enough if we show that

$$
P\left(X\left(t_{k}+a_{t_{k}}\right)-X\left(t_{k}\right) \leq\left(\varepsilon^{*}-\varepsilon_{1}\right) \beta\left(t_{k}\right) \text { i.o }\right)=0 .
$$

We know that $t^{-\frac{1}{\alpha}} X(t) \stackrel{d}{=} X(1)$ which implies

$$
P\left(X\left(t_{k}+a_{t_{k}}\right)-X\left(t_{k}\right) \leq\left(\varepsilon^{*}-\varepsilon_{1}\right) \beta\left(t_{k}\right)\right)=P\left(X(1) \leq \frac{\left(\varepsilon^{*}-\varepsilon_{1}\right) \beta\left(t_{k}\right)}{a_{t_{k}}^{\frac{1}{\alpha}}}\right)
$$

and

$$
\frac{\left(\varepsilon^{*}-\varepsilon_{1}\right) \beta\left(t_{k}\right)}{a_{t_{k}}^{\frac{1}{\alpha}}} \simeq\left(\varepsilon^{*}-\varepsilon_{1}\right) \theta_{\alpha}\left(\log \left(\frac{t_{k}}{a_{t_{k}}} \log t_{k}\right)\right)^{\frac{\alpha-1}{\alpha}} .
$$

By taking $x=\left(\varepsilon^{*}-\varepsilon_{1}\right) \theta_{\alpha}\left(\log \frac{t_{k}}{a_{t_{k}}} \log t_{k}\right)^{\frac{\alpha-1}{\alpha}}$, where

$$
g\left(t_{k}\right)=\frac{t_{k}}{a_{t_{k}}} \log t_{k},
$$

in the above lemma, one can find a $k_{4}(>0)$ and $C_{2} \quad(>0)$ such that for all $k \geq k_{4}$,

$$
P\left(X(1) \leq \frac{\left(\varepsilon^{*}-\varepsilon_{1}\right) \beta\left(t_{k}\right)}{a_{t_{k}}^{\frac{1}{\alpha}}}\right) \leq \frac{C_{2}}{\left(\log g\left(t_{k}\right)\right)^{\frac{1}{2}}} \exp \left\{-\left(\varepsilon^{*}-\varepsilon_{1}\right)^{\frac{\alpha}{\alpha-1}} \log g\left(t_{k}\right)\right\} .
$$

Observe that using properties of $\left\{a_{t}\right\}$ one can find some constant $C_{3}\left(>C_{2}\right)$ and $k_{4}$ such that for all $k \geq k_{4}$, we have,

$$
P\left(X(1) \leq \frac{\left(\varepsilon^{*}-\varepsilon_{1}\right) \beta\left(t_{k}\right)}{a_{t_{k}}^{\frac{1}{\alpha}}}\right) \leq \frac{C_{3}}{\left(g\left(t_{k}\right)\right)^{\left(\varepsilon^{*}-\varepsilon_{1}\right)^{\frac{\alpha}{\alpha-1}}}} .
$$

Notice that $\varepsilon^{*}=\inf \left\{\varepsilon>0: \sum_{k=k_{0}}^{\infty}\left(g\left(t_{k}\right)\right)^{-\varepsilon^{-\lambda}}<\infty\right\}$ and $\lambda=\frac{\alpha}{\alpha-1}<0, \quad 0<\alpha<1$ which yields $\varepsilon^{*} \geq 1$. 
Since $\varepsilon_{1} \in(0,1)$, choose $\varepsilon_{1}$ sufficiently small one can find $k_{5}\left(\geq k_{4}\right)$ such that for all $k \geq k_{5}$,

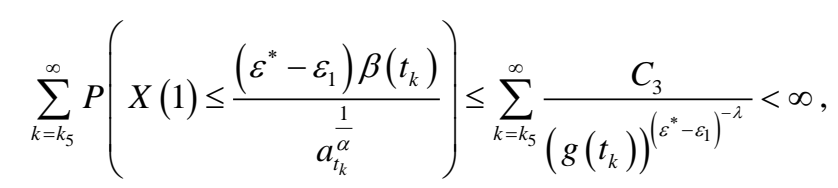

where $\lambda=\frac{\alpha}{\alpha-1}, 0<\alpha<1$.

By Borel-Cantelli lemma, (2.6) holds which implies (2.3) holds and proof of the theorem is completed.

\section{Example 1}

Let $t_{k}=\exp \left\{k^{p}\right\}, \quad 0<p<1, k \geq 1$ and $a_{t_{k}}=\left[p t_{k}\right]$. Observe that condition (2.1) is satisfied and hence we have $\varepsilon^{*}=p^{(1-\alpha) / \alpha}$.

\section{Theorem 2.2}

Let $a_{t}, t>0$, be a non-decreasing function of $t$ such that 1) $\left.0<a_{t} \leq t, 2\right) a_{t} \rightarrow \infty$ as $t \rightarrow \infty$; and 3) $\frac{a_{t}}{t} \downarrow 0$ as $t \rightarrow \infty$. Let $\left(t_{k}\right)$ be an increasing sequence of positive integers such that

$$
\operatorname{Liminf}_{k \rightarrow \infty} \frac{t_{k+1}-t_{k}}{a_{t_{k}}}>1
$$

Then

$$
\operatorname{Liminf}_{k \rightarrow \infty} \frac{Y\left(t_{k}\right)}{\beta\left(t_{k}\right)}=1 \text { a.s. }
$$

\section{Proof}

To prove the theorem, it is enough to show that for any $\varepsilon \in(0,1)$,

$$
P\left(Y\left(t_{k}\right) \leq(1+\varepsilon) \beta\left(t_{k}\right) \text { i.o }\right)=1
$$

and

$$
P\left(Y\left(t_{k}\right) \leq(1-\varepsilon) \beta\left(t_{k}\right) \text { i.o }\right)=0
$$

By the theorem of Vasudeva and Divanji [7], we claim that $\operatorname{Liminf}_{k \rightarrow \infty} \frac{Y\left(t_{k}\right)}{\beta\left(t_{k}\right)} \geq \operatorname{Liminf}_{t \rightarrow \infty} \frac{Y(t)}{\beta(t)}=1$ a.s., which establishes (2.10).

The condition (2.8), i.e., $\operatorname{Liminf}_{k \rightarrow \infty} \frac{t_{k+1}-t_{k}}{a_{t_{k}}}>1$ implies that there exists a $k_{1}$ such that $t_{k+1}>t_{k}+a_{t_{k}}$, for all $k \geq k_{1}$. This in turn implies that $\left\{Y\left(t_{k}\right), k \geq 1\right\}$ is a sequence of mutually independent r.v.s. We can observe that with a minor modification, the proof of (2.9) follows on similar lines of (2.3). That is using Lemma, one can find $C_{1}$ and $k_{2}$ such that for all $k \geq k_{2}$,

$$
P\left(X(1) \leq \frac{\left(1+\varepsilon_{1}\right) \beta\left(t_{k}\right)}{a_{t_{k}}^{\frac{1}{\alpha}}}\right) \geq \frac{C_{1}}{\left(\log g\left(t_{k}\right)\right)^{\frac{1}{2}}\left(g\left(t_{k}\right)\right)^{\left(1+\varepsilon_{1}\right)^{\frac{\alpha}{\alpha-1}}} .}
$$

Choose $\varepsilon_{4}(>0)$ such that $\left(1+\varepsilon_{1}\right)^{\frac{\alpha}{\alpha-1}}<\left(1-\varepsilon_{4}\right)<1$ and hence we have,

$$
P\left(X(1) \leq \frac{\left(1+\varepsilon_{1}\right) \beta\left(t_{k}\right)}{a_{t_{k}}^{\frac{1}{\alpha}}}\right) \geq \frac{C_{1}}{\left(\log g\left(t_{k}\right)\right)^{\frac{1}{2}}\left(g\left(t_{k}\right)\right)^{\left(1-\varepsilon_{4}\right)}} .
$$


Following similar arguments of proof of (2.4) and (2.5), we get $\sum_{k=k_{2}}^{\infty} P\left(Y\left(t_{k}\right) \leq\left(1+\varepsilon_{1}\right) \beta\left(t_{k}\right)\right)=\infty$, which in turn implies the proof of (2.9). Hence the proof of the theorem is completed.

\section{Example 2}

Let $t_{k}=\exp \left\{k^{p}\right\}, \quad p>1, \quad k \geq 1$ and $a_{t_{k}}=\frac{t_{k}}{\log \log t_{k}}$, then condition (2.8) is satisfied and hence we have the result.

\section{Remark 1}

Observe that for any $t>0$, the dividing term $\beta(t)$ changes with $a_{t}$ in the limit inferior of increments of stable subordinators whereas for stable subordinators the dividing term does not change with $a_{t}$ (See Vasudeva and Divanji [7]). However it is interesting to note that for increasing subsequences $\left\{t_{k}, k \geq 1\right\}$ of $\{t\}$, the form of dividing constant changes with $a_{t_{k}}$ for both increments of stable subordinators (Theorems 2.1 and 2.2) and stable subordinators (See Mijnheer [5]).

\section{Parallel Results for Delayed Sums and Existence of Moments for Boundary Crossing r.v.s}

Let $\left\{X_{n}, n \geq 1\right\}$ be a sequence of i.i.d strictly positive stable r.v.s with index $\alpha, 0<\alpha<1$. Let $\left\{a_{n}, n \geq 0\right\}$ be a sequence of non-decreasing functions of positive integers of $n$ such that $0<a_{n} \leq n$, for all $n$ and we assume that $\frac{a_{n}}{n} \downarrow 0$ as $n \rightarrow \infty$. Define $\beta(n)=\theta_{\alpha} a_{n}^{\frac{1}{\alpha}}\left(\log \frac{n}{a_{n}}+\log \log n\right)^{\frac{\alpha-1}{\alpha}}$, where $\theta_{\alpha}=(B(\alpha))^{\frac{1-\alpha}{\alpha}}$ and $B(\alpha)=(1-\alpha) \alpha^{\frac{\alpha}{1-\alpha}}\left(\cos \left(\frac{\pi \alpha}{2}\right)\right)^{\frac{1}{\alpha-1}}$, where $0<\alpha<1$. Observe that the process has the property that $n^{-\frac{1}{\alpha}} X(n)$ and $X(1)$ are identically distributed. Let $S_{n}=\sum_{k=1}^{n} X_{k}$ and set $M_{n}=S_{n+a_{n}}-S_{n}$, where $\left\{M_{n}, n \geq 1\right\}$ is called a (forward) delayed sum (See Lai [3]). Define the r.v.s, $X_{n}=X(n)-X(n-1)$, $n=1,2, \cdots \quad ; \quad X(0)=0 \quad$, then $\quad S_{n}=\sum_{k=1}^{n} X_{k}=X_{n} \quad$ with $\quad S_{0}=0 \quad$, which yields $M_{n}=S_{n+a_{n}}-S_{n}=X\left(n+a_{n}\right)-X(n)=Y(n)$.

Now we extend the Theorems 2.1 and Theorem 2.2 to $\left\{M_{n}, n \geq 1\right\}$ under the subsequence principle.

\section{Theorem 3.1}

Let $\left\{a_{n}, n>0\right\}$ be a sequence of non-decreasing functions of positive integers of $n$ such that 1) $0<a_{n} \leq n, n>0$, 2) $a_{n} \rightarrow \infty$, as $n \rightarrow \infty$, and 3) $\frac{a_{n}}{n} \downarrow 0$ as $n \rightarrow \infty$. Let $\left(n_{k}, k \geq 1\right)$ be an increasing sequence of positive integers such that $\operatorname{Limsup}_{k \rightarrow \infty} \frac{n_{k+1}-n_{k}}{a_{t_{k}}}<\infty$. Then $\operatorname{Liminf}_{k \rightarrow \infty} \frac{M_{n_{k}}}{\beta\left(n_{k}\right)}=\varepsilon^{*}$ a.s, where

$$
\varepsilon^{*}=\inf \left\{\varepsilon>0: \sum_{k=k_{0}}^{\infty}\left(g\left(n_{k}\right)\right)^{-\varepsilon^{-\lambda}}<\infty\right\} \text { and } \lambda=\frac{\alpha}{\alpha-1}<0,0<\alpha<1 \text {. }
$$

\section{Proof}

To prove the theorem it is sufficient to show that for any given $\varepsilon_{2} \in(0,1)$,

$$
P\left(M_{n_{k}} \leq\left(\varepsilon^{*}+\varepsilon_{2}\right) \beta\left(n_{k}\right) \text { i.o }\right)=1
$$

and

$$
P\left(M_{n_{k}} \leq\left(\varepsilon^{*}-\varepsilon_{2}\right) \beta\left(n_{k}\right) \text { i.o }\right)=0
$$


The proof of (3.2) follows on the similar lines of Vasudeva and Divanji [7] and the proof of (3.1) is an immediate consequence of (2.2). Hence the details are omitted.

\section{Theorem 3.2}

Let $\left\{a_{n}, n>0\right\}$ be a sequence of non-decreasing functions of positive integers of $n$ such that 1) $\left.0<a_{n} \leq n, \quad n>0,2\right) \quad a_{n} \rightarrow \infty$, as $n \rightarrow \infty$, and 3) $\left(a_{n} / n\right) \downarrow 0$ as $n \rightarrow \infty$. Let $\left(n_{k}, k \geq 1\right)$ be an increasing sequence of positive integers such that $\operatorname{Liminf}_{k \rightarrow \infty} \frac{n_{k+1}-n_{k}}{a_{n_{k}}}>1$. Then $\operatorname{Liminf}_{k \rightarrow \infty} \frac{M_{n_{k}}}{\beta\left(n_{k}\right)}=1$ a.s.

\section{Proof}

The proof of the theorem is a direct consequence of above Theorem 2.2 and hence the details are omitted.

In association with the LIL or strong laws the study of the r.v. giving the number of boundary crossings has become very significant, since this study gives the precision of the LIL or Strong laws. Here we study the existence of moments for boundary crossing random variables related to Theorems 2.1, 2.2, 3.1 and 3.2 .

Define, for any $\delta>0$,

where

$$
Z_{t_{k}}(\delta)=\left\{\begin{array}{l}
1, \text { if } Y\left(t_{k}\right) \leq(\theta-\delta) \beta\left(t_{k}\right) \\
0, \text { otherwise }
\end{array}\right.
$$

$$
\begin{gathered}
\theta=\left\{\begin{array}{l}
\varepsilon^{*}, \text { if }\left(t_{k}\right) \text { is at least geometrically fast } \\
1, \text { if }\left(t_{k}\right) \text { is at most geometrically fast }
\end{array}\right. \\
\varepsilon^{*}=\inf \left\{\varepsilon_{1}>0: \sum_{k=k_{0}}^{\infty}\left(g\left(t_{k}\right)\right)^{-\varepsilon_{1}^{-\lambda}}<\infty\right\}, \\
g\left(t_{k}\right)=\left(\frac{t_{k}}{a_{t_{k}}} \log t_{k}\right)
\end{gathered}
$$

and $\lambda=\frac{\alpha}{\alpha-1}<0,0<\alpha<1$. For any $\delta>0$, we know that from (2.3) and (2.10) we have

$$
P\left(Y\left(t_{k}\right) \leq(\theta-\delta) \beta\left(t_{k}\right) \text { i.o }\right)=0 .
$$

Define $N_{\infty}(\delta)=\sum_{k=1}^{\infty} Z\left(t_{k}\right)$ and observe that $N_{\infty}(\delta)$ is a proper r.v in view of (3.3). Let $\left(N_{m_{k}}(\delta)\right)$ be the corresponding sequence of partial Sums. i.e., $N_{m_{k}}(\delta)=\sum_{k=1}^{m_{k}} Z\left(t_{k}\right)$. If $N_{\infty}(\delta)=\sum_{k=1}^{\infty} Z\left(t_{k}\right)$, we know that $P\left(N_{\infty}(\delta)<\infty\right)=1$ or $N_{\infty}(\delta)$ is a proper r.v or number of boundary crossings and hence it is interesting to study the existence of moments for this boundary crossing r.v.s and obtain moments of this proper r.v. $N_{\infty}(\delta)$ as Corollaries to Theorems 2.1, 2.2, 3.1 and 3.2. This boundary crossing r.v.s $N_{\infty}(\delta)$ was studied by various authors like Slivka, J. [8] and Slivka, J and Savero, N.C. [9]. Here we show that all the moments in $0<\eta \leq 1$ are finite for $N_{\infty}(\delta)$.

Corollary 3.1: Let $\{Y(t)\}$ and $\left\{a_{t}\right\}$ are defined in introduction. Let $\left(t_{k}\right)$ be an increasing sequence of positive integers. Then for $\delta>0$ and for any $\eta, 0<\eta \leq 1$,

$$
E N_{\infty}^{\eta}<\infty, \text { if } \sum_{k=1}^{\infty} t_{k}^{\eta-1} P\left(Y\left(t_{k}\right) \leq(\theta-\delta) \beta\left(t_{k}\right)\right)<\infty
$$

Proof: First we show that, for $\eta=1, E N_{\infty}(\delta)<\infty$, and then claim that the existence of lower moments follows from the higher moments. Observe that $E N_{\infty}(\delta)=\sum_{k=1}^{\infty} P\left(Y\left(t_{k}\right) \leq(\theta-\delta) \beta\left(t_{k}\right)\right)$. Following similar lines of the proof of (2.3) and (2.10), we can find some constant $C_{1}>0$ and some $k_{1}>0$ such that, for all $k \geq k_{1}$, 


$$
E N_{\infty}(\delta) \leq C_{1} \sum_{k=k_{1}}^{\infty} \frac{1}{\left(g\left(t_{k}\right)\right)^{(\theta-\delta)^{-\lambda}}}<\infty, \lambda=\frac{\alpha}{\alpha-1}<0,0<\alpha<1 .
$$

Convergence of the series follows from the definition of $\theta$ and proofs of (2.3) and (2.10). Consequently, $E N_{\infty}(\delta)<\infty$ for $\eta=1$, and therefore $E N_{\infty}^{\eta}<\infty$, for $\eta<1$. This completes the proof of the Corollary.

Corollary 3.2: Let $\left\{M_{n}\right\}$ and $\left\{a_{n}\right\}$ are defined in Section 3 above. Let $\left(n_{k}\right)$ be an increasing sequence of positive integers. Then for $\delta>0$ and for any $\eta, 0<\eta \leq 1$,

$$
E N_{\infty}^{\eta}<\infty \text {, if } \sum_{k=1}^{\infty} n_{k}^{\eta-1} P\left(M_{n_{k}} \leq(\theta-\delta) \beta\left(n_{k}\right)\right)<\infty .
$$

Proof: Proof follows on similar lines of Corollary 3.1 and we omit the details.

\section{Remark 2}

Stable subordinators are heavy tailed processes and our results are expected to be useful in Business, Insurance and so on. For example in share market, determination of prices of shares, fluctuations exceeding a certain level in SENSEX have major role to play in determining the share value and are to be predicted by considering extreme items.

\section{Acknowledgements}

The authors wish to thanks the anonymous referee for his valuable comments. Also authors thank Professor M. Sreehari, M. S. University, Baroda, Gujarath, whose constant encouragement in this field of research. The first author wishes to express his sincere thanks to University Grants Commission, New Delhi, for financial support in the form of Major Research Project (F. No: 34-156/2008 (SR)). Also the second author acknowledge with thanks, the financial assistance from the University Grant Commission (UGC), New Delhi, India as in the form of Research Fellowship in Sciences for Meritorious Students.

\section{References}

[1] Gut, A. (1986) Law of Iterated Logarithm for Subsequences. Probability and Mathematical Statistics, 7, 27-58.

[2] Hwang, K.S., Choi, Y.K. and Jung, J.S. (1997) On Superior Limits for the Increments of Gaussian Processes. Statistics and Probability Letter, 35, 289-296. http://dx.doi.org/10.1016/S0167-7152(97)00025-4

[3] Lai, T.L. (1973) Limit Theorems for Delayed Sums. The Annals of Probability, 2, 432-440. http://dx.doi.org/10.1214/aop/1176996658

[4] Mijhneer, J.L. (1975) Sample Path Properties of Stable Process. Mathematisch, Amsterdam.

[5] Mijnheer, J.L. (1995) On the Law of Iterated Logarithm for Subsequences for a Stable Subordinator. Journal of Mathematical Sciences, 76, 2283-2286. http://dx.doi.org/10.1007/BF02362699

[6] Schwabe, R. and Gut, A. (1996) On the Law of the Iterated Logarithm for Rapidly Increasing Subsequences. Mathematische Nachrichten, 178, 309-332. http://dx.doi.org/10.1002/mana.19961780115

[7] Vasudeva, R. and Divanji, G. (1988) Law of Iterated Logarithm for the Increments of Stable Subordinators. Stochastic Processes and Their Applications, 28, 293-300. http://dx.doi.org/10.1016/0304-4149(88)90102-0

[8] Slivka, J. (1969) On the LIL. Proceedings of the National Academy of Sciences of the United States of America, 63, 2389-291.

[9] Slivka, J. and Savero, N.C. (1970) On the Strong Law of Large Numbers. Proceedings of the American Mathematical Society, 24, 729-734. 